\title{
SALT AND PEPPER NOISE REDUCTION AND EDGE DETECTION ALGORITHM BASED ON NEUTROSOPHIC LOGIC
}

\footnotetext{
Abstract

Noise reduction of images is a challenging task in image processing. Salt and pepper noise is one kind of noise that affects a gray-scale image significantly. Generally, the median filter is used to reduce salt and pepper noise; it gives optimum results while compared to other image filters. Median filter works only up to a certain level of noise intensity. Here we proposed a neighborhoodbased image filter called nbd-filter, it works perfectly for gray image regardless of noise intensity. It reduces salt and pepper noise significantly at any noise level and produces a noise-free image. Further, we proposed an edge detection algorithm based on the neutrosophic set, it detects edges efficiently for images corrupted by noise and noise-free images. Neutrosophic set (NS) is a powerful tool to deal with indeterminacy. Since most of the real-life images consists of indeterminate regions, Neutrosophy is a perfect tool for edge detection. In this paper, the neutrosophic set is applied to the image domain and a novel edge detection technique is proposed.

Keywords neutrosophic set, digital image processing, image analysis, image denoising, edge detection

Citation

Copyright 


\section{Introduction}

Digital images play a significant role in many scientific fields such as satellite television, artificial intelligence, robotics and automation, signature validation, computer resonance imaging, geographical information systems and astronomy, and almost every day-to-day life. Image denoising is a challenging task in every field deal with digital images. Digital images can be corrupted by various types of noise. Image noise not only deteriorates the quality of images but also makes difficult to perform image analysis such as image segmentation, edge detection, etc. Salt and pepper noise is one of the types of noise which affects digital images significantly. This type of noise may occur due to error transmission and acquisition of the image. Salt and pepper noise corrupt pixels of digital image only by either minimum gray level intensity or maximum gray level intensity (i.e. corrupted by 0 and 255 in 8-bit image).

The main goal of our proposed filter (nbd-filter) is to reduce the maximum noise level, at the same time preserve image information as much as possible. Followed by nbd-filter, we proposed a novel edge detection technique based on the neutrosophic set. Merit of our edge detection technique is, it is neutrosophic based algorithm and works much better than most of the other conventional techniques. Section 2 consists the state of the art review study. Here, the previous works on image denoising and edge detection methods are discussed. Section 3 consists main theme of the proposed work. A neighborhood based image denoising filter (nbd-filter) to reduce salt and pepper noise is proposed. Since the proposed filter lonely based on the concept of neighborhoods, it is user-friendly and very efficient. Followed by nbd-filter, a novel edge detection technique based on neutrosophic set is proposed. Images denoised by the proposed filter are taken for edge detection. The proposed edge detector performs very well on denoised images as well as noise-free images. Section 4 consists experimental results of the proposed denoising filter and the proposed edge detector. Further, we analyze the performance of these techniques by popular metrics Peak Signal to Noise Ratio (PSNR), Mean Square Error (MSE) and Figure of Matric (FoM). Section 5 concludes the proposed work.

\section{State of the art review}

Image processing becomes more critical whenever an image is corrupted by noise. Salt and pepper noise is one of the noises that affect grayscale images. It is the random distribution of minimum gray level and maximum gray level to image pixels. For a grayscale image, there are various linear and non-linear image filters such as mean filter, median filter, wiener filter, and Gaussian filter, etc. In general Wiener filter gives optimum results for all types of noises, but only at the minimum noise level. In most of the situations, the median filter gives optimum result for salt and pepper noise. But the median filter gives the best results only up to a certain noise level. For high intensity noise level, the median filter perform less effective. The proposed filter reduce salt and pepper noise significantly at any noise level, unlike 
any other filters. In 2011, Yanhui Guo et al. [5] proposed a novel image denoisng technique based on neutrosophic logic. Hongjin Ma et al. [7] proposed a two stage filter to reduce salt and pepper noise based on adaptive directional mean filter. Raza et al. [10] proposed Decision based partially trimmed global mean filter (PTGMF) to reduce high intensity salt and pepper noise. Sathua et al. [11] proposed a denoising technique to remove Salt and pepper noise in gray-scale and color image based on neighborhood concepts. Several denoising methods based on neutrosophic set have been proposed to reduce salt and pepper noise, Gaussian noise, speckle noise $[1,8]$.

Neutrosophy deals with origin, nature, and scope of neutralities, as well as their interactions with different ideational spectra. Neutrosophy is the basis of neutrosophic sets (derivative of neutrosophy). Neutrosophy is one of the best tools to deal with indeterminacy by defining membership values. Neutrosophic set consist of three parameters such as truth-membership degree, indeterminate-membership degree, and falsify-membership degree. Guo et al. [5] applied the neutrosophic set to the image domain and developed some image denoising concepts. Neutrosophy is a branch of philosophy which was introduced by Florentin Samarandache [13-15]. Since neutrosophy can deal with indeterminacy, it is suitable tool for image analysis. In 2008, H.D. Cheng et al. [3] introduced the representation of image in neutrosophic domain. Guo et al. [6] proposed edge detection algorithm based on neutrosophic set in 2014 . Esar Sert et al. [12] proposed image edge detection technique combined with ChanVase algorithm in 2019. Proposed edge detection technique perform very well for images corrupted by noise as well as noise-free images. Further, we compare the performance of proposed technique with most popular edge detection techniques through Figure of Merit(FoM) metric which was defined by William K Pratt [9] in 2013. Guo's concept of an image in the neutrosophic domain is given below.

\subsection{Neutrosophic image}

Neutrosophic set consists of three parameters as truth-membership, indeterminacy, and falsify-membership, unlike other types of fuzzy sets. The classical set contains only truth and false memberships. But an image might contain indeterminate regions; classical set cannot interpret indeterminacy. So neutrosophic set can handle indeterminate regions in images. Neutrosophy has a wide range of applications in science and engineering.

In 2008, H.D Cheng and Yanhui Guo [3,4] introduced the representation of a grayscale image in the neutrosophic domain. A neutrosophic image $P_{N S}$ is characterized by three subsets $T, I$ and $F$.

A pixel $P$ in an image is described as $P(T, I, F)$ and belongs to $W$ in the following way: it is $t \%$ true, $i \%$ indeterminate and $f \%$ false in the bright pixel set, where $t$ varies in $T, i$ varies in $I$ and $f$ varies in $F$. Each component has a value in $[0,1]$.

We define a $3 \times 3$ neighborhood window $W$ for each pixels. Pixel $P(i, j)$ in the image domain is transformed into neutrosophic domain $P_{N S}(i, j)=$ $T(i, j), I(i, j), F(i, j)$, where $T(i, j), I(i, j)$ and $F(i, j)$ represents membership degrees 
of white pixel set, indeterminate pixel set and non-white pixel set, respectively, which are defined as:

$$
\begin{array}{r}
T(i, j)=\frac{\bar{g}(i, j)-\bar{g}_{\text {min }}}{\bar{g}_{\text {max }}-\bar{g}_{\text {min }}} \\
I(i, j)=\frac{\delta(i, j)-\delta_{\text {min }}}{\delta_{\text {max }}-\delta_{\text {min }}} \\
F(i, j)=1-T(i, j)=\frac{\bar{g}_{\text {max }}-\bar{g}(i, j)}{\bar{g}_{\text {max }}-\bar{g}_{\text {min }}}
\end{array}
$$

where $\bar{g}(i, j)$ represents mean intensity of pixel in some neighborhoods in $W$. Here:

$$
\begin{array}{r}
\bar{g}(i, j)=\frac{1}{W \times W} \sum_{m=i-w / 2}^{i+w / 2} \sum_{n=j-w / 2}^{j+w / 2} g(m, n) \\
\delta(i, j)=|g(i, j)-\bar{g}(i, j)| \\
\delta_{\max }=\max \delta(i, j) \quad \delta_{\min }=\min \delta(i, j)
\end{array}
$$

\section{Proposed image denoising and edge detection techniques}

\subsection{Image denoising algorithm}

A new image denoising technique based on the concepts of neighborhood is proposed. Sathua's [11] technique deals with dynamic neighborhood window size. It means, window size depends on the noise intensity level. Removal of high intensity noise requires large neighborhood window and vice versa. Due to larger neighborhoods, the processing time is high for high resolution image. Also, by using larger neighborhood window, sometimes the corrupted pixels are replaced by unmatched pixels (i.e. neighborhood pixels which has significant deviation from original pixel). To overcome these difficulties, we proposed nbd-filter which has a constant $3 \times 3$ neighborhood window for any noise variance. In this technique, we define a $3 \times 3$ neighborhood around each pixel of a given noisy image.

We define the proposed filter in two stages and one additional stage for maximum noise variance. Salt and pepper noise is the randomly distributed corrupted pixels of minimum gray level (0) and maximum gray level (255) in an image. We reduce these corrupted pixels in three stages. We used MATLAB version 2013a for our experimental results.

For our convenience, image floating type uint is changed into double (i.e. pixel intensity values ranges from 0 to 1 with minimum of 0 pixel value and maximum of 1 pixel value).

\section{Stage 1}

In stage 1 , we restore pixels corrupted by high noise intensity (i.e. pixels have value 1 ). Consider $g$ be a grayscale image and $g(i, j)$ is a pixel value at the location $i, j$. Let $w$ be $3 \times 3$ neighborhood window of each pixel $g(i, j)$ and $V_{\alpha}$ be the set of uncorrupted neighborhoods of each pixel $g(i, j)$. 
Then, execute the following formula:

$$
G_{\alpha}(i, j)=\left\{\begin{array}{lc}
g_{V_{\alpha}}(i, j) & \text { if } g(i, j)=1 \\
g(i, j) & \text { otherwise }
\end{array}\right.
$$

where

$$
g_{V_{\alpha}}(i, j)=\operatorname{median}\left\{\operatorname{median}\left\{V_{\alpha}(m, n)\right\}_{n=j-w / 2}^{j+w / 2}\right\}_{m=i-w / 2}^{i+w / 2}
$$

(i.e. The median pixel value of each uncorrupted neighborhood set). From the above equation, we conclude that $\max \left\{G_{\alpha}(i, j)\right\} \neq 1$ and $\min \left\{G_{\alpha}(i, j)\right\} \equiv 0$ (minimum value is zero because of salt and pepper noise). In Matlab, we execute the above formula iteratively (2-iterations).

\section{Stage 2}

In stage 2 , we restore pixels corrupted by low noise intensity (i.e. zero pixels) as much as possible. Define $3 \times 3$ neighborhoods for each pixels of $G_{\alpha}$. Let $w$ be the $3 \times 3$ neighborhood window and $V_{\beta}$ be the uncorrupted neighborhoods pf each pixels of $G_{\alpha}(i, j)$ (i.e. pixel values other than 0 and 1 ). The second stage denoised image is defined by:

$$
G_{\beta}(i, j)=\left\{\begin{array}{lc}
g_{V_{\beta}}(i, j) & \text { if } G_{\alpha}(i, j)=0 \\
G_{\alpha}(i, j) & \text { otherwise }
\end{array}\right.
$$

where

$$
g_{V_{\beta}}(i, j)=\operatorname{median}\left\{\operatorname{median}\left\{V_{\beta}(m, n)\right\}_{n=j-w / 2}^{j+w / 2}\right\}_{m=i-w / 2}^{i+w / 2}
$$

The above formula is executed iteratively to get better result.

\section{Stage 3 (Additional Stage)}

In this stage, we will restore original image as much as possible. In stages 1 and 2 almost all corrupted pixels are restored. But, in some cases, due to high noise intensity, some pixels have only corrupted neighborhoods (i.e. only pixels 0's and 1's in the neighborhood). Those pixels were not processed in the previous stages and displayed as 'NaN' in MATLAB. Let $V_{E n}$ be the set of valid pixels (i.e. pixels other than $\mathrm{NaN}$ ).

We execute the following formula to restore such pixels:

$$
G_{E n}(i, j)=\left\{\begin{array}{lc}
g_{V_{E n}}(i, j) & \text { if } G_{\beta}(i, j)=N a N \\
G_{\beta}(i, j) & \text { otherwise }
\end{array}\right.
$$


where:

$$
g_{V_{E n}}(i, j)=\operatorname{median}\left\{\operatorname{median}\left\{V_{E n}(m, n)\right\}_{n=j-w / 2}^{j+w / 2}\right\}_{m=i-w / 2}^{i+w / 2}
$$

The above formula is executed certain number of iterations to restore all 'NaN' pixels. The sufficient number of iterations are directly proportional to noise variance. It means, high noise intensity requires, maximum number of iterations and vice versa. However, at the maximum of 25 iterations are sufficient to restore corrupted pixels. Our MATLAB algorithm defined such that it executes sufficient number of iterations automatically.

\subsection{Edge detection algorithm}

Followed by image denoising, we proposed edge detection technique based on neutrosophic logic. The image processed by nbd-filter is taken for edge detection. However, the proposed edge detection technique is also suitable for default noise-free images. Our edge detection algorithm has the following steps.

\section{Step 1}

Convert the image $G_{E n}(i, j)$ (denoised image or default noise-free image) into the neutrosophic domain using the neutrosophic image concept which is given in Section 2.1.

\section{Step 2}

Compute the local maximum value for each pixel of an image by taking the neighborhood window $(w)$. Here we take $3 \times 3$ neighborhood and set $w=3$.

The local maximum value is given by:

$$
\hat{g}(i, j)=\max \left\{\max \{g(m, n)\}_{n=j-w / 2}^{j+w / 2}\right\}_{m=i-w / 2}^{i+w / 2}
$$

\section{Step 3}

Replace local mean value in our neutrosophic domain by local maximum value. Then we have the following set of equations.

$$
\begin{array}{r}
\hat{T}(i, j)=\frac{\hat{g}(i, j)-\hat{g}_{\text {min }}}{\hat{g}_{\text {max }}-\hat{g}_{\text {min }}} \\
\hat{I}(i, j)=\frac{\hat{\delta}(i, j)-\hat{\delta}_{\text {min }}}{\hat{\delta}_{\text {max }}-\hat{\delta}_{\text {min }}} \\
\hat{F}(i, j)=1-\hat{T}(i, j)=\frac{\hat{g}_{\text {max }}-\hat{g}(i, j)}{\hat{g}_{\text {max }}-\hat{g}_{\text {min }}}
\end{array}
$$




$$
\hat{\delta}(i, j)=|g(i, j)-\hat{g}(i, j)| \quad \hat{\delta}_{\max }=\max \{\hat{\delta}(i, j)\} \quad \hat{\delta}_{\min }=\min \{\hat{\delta}(i, j)\}
$$

where $\hat{g}(i, j)$ represents local maximum intensity of pixel in some neighborhood $w$. Also $\hat{\delta}(i, j)$ represents the absolute value of difference between the intensity value and local maximum intensity value of each pixels.

\section{Step 4}

Compute the gradient value of the truth image $\hat{T}(i, j)$ for each pixels and is denoted by $\nabla \hat{T}(i, j)$.

\section{Step 5}

Define $3 \times 3$ neighborhood $W$ of $\hat{\delta}(i, j)$ and optimum threshold value $\gamma$ (Here, we take $\gamma=0.2$ ). Also compute maximum and mean value of each neighborhoods $W$.

$$
\begin{gathered}
W=\left\{\{\nabla \hat{T}(i, j)\}_{n=j-w / 2}^{j+w / 2}\right\}_{m=i-w / 2}^{i+w / 2} \\
W_{\text {max }}=\operatorname{maximum}\{W\} \quad ; \quad W_{\text {mean }}=\operatorname{mean}\{W\}
\end{gathered}
$$

\section{Step 6}

Define

$V^{+}=$Set of pixels which are greater than $\gamma$ in each neighborhood.

$V^{-}=$Set of pixels which are less than $\gamma$ in each neighborhood.

$N(W)=$ Number of pixels in a neighborhood $W$.

$N\left(V^{+}\right)=$Number of pixels in the set $V^{+}$.

$N\left(V^{-}\right)=$Number of pixels in the set $V^{-}$.

\section{Step 7}

Finally, the proposed edge image $E_{N S}(i, j)$ is given by:

$$
E_{N S}(i, j)= \begin{cases}1 \quad \text { if } \quad & \left.N\left(V^{+}\right) \geq N\left(V^{-}\right) \& \nabla \hat{T}(i, j)=W_{\text {max }} \quad \text { (or }\right) \\ & N\left(V^{+}\right) \geq N\left(V^{-}\right) \& \nabla \hat{T}(i, j)=W_{\text {mean }} \\ 0 & \text { otherwise }\end{cases}
$$

\section{Experimental results and discussions}

\subsection{Image denoising experimental results}

Figure 1 consists denoised images by various image filters and proposed filter. Proposed filter has higher PSNR values and lower MSE values at different noise levels. It means the accuracy of nbd-filter is maximum over other image filters for salt and pepper noise. 


\begin{tabular}{cllll}
\hline Noisy image & Wiener filter & Median filter & Sathua's filter & nbd filter \\
\hline
\end{tabular}

a)

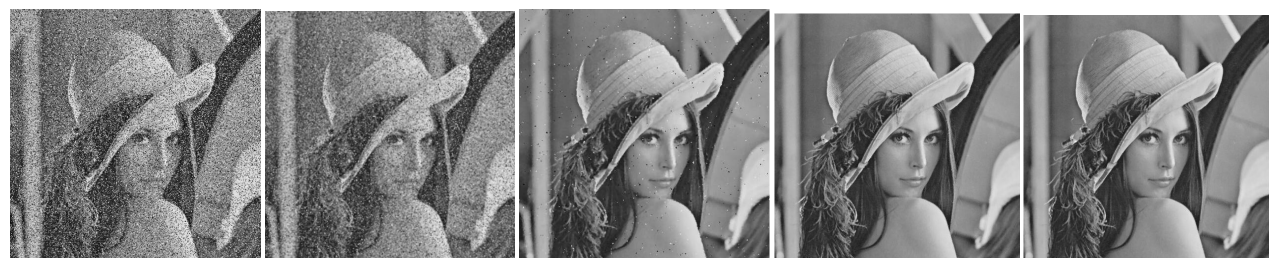

b)
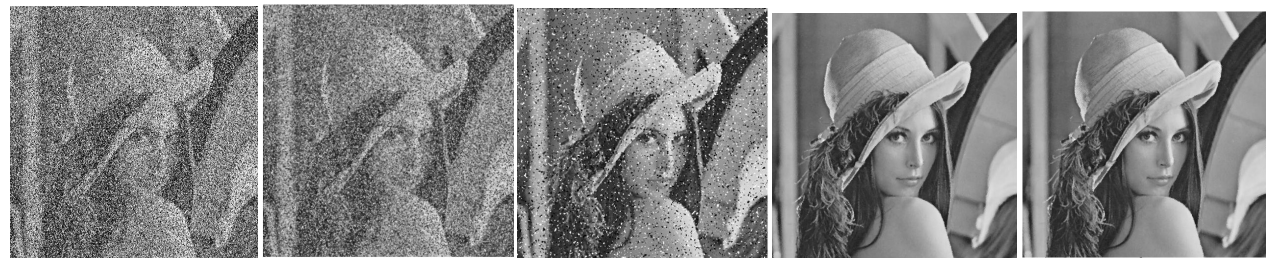

c)
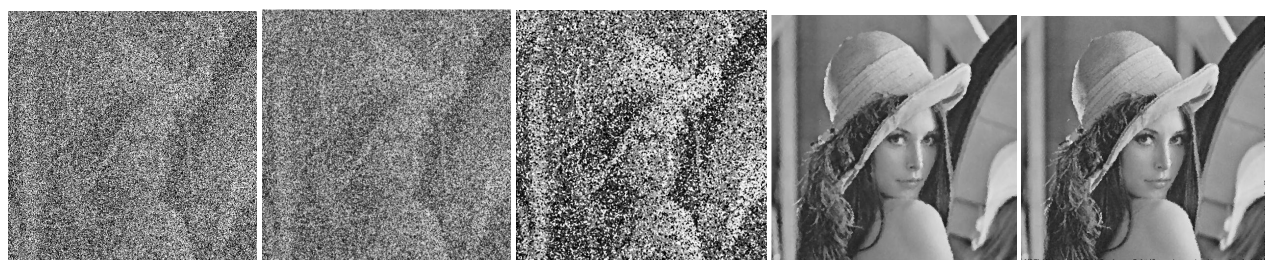

d)
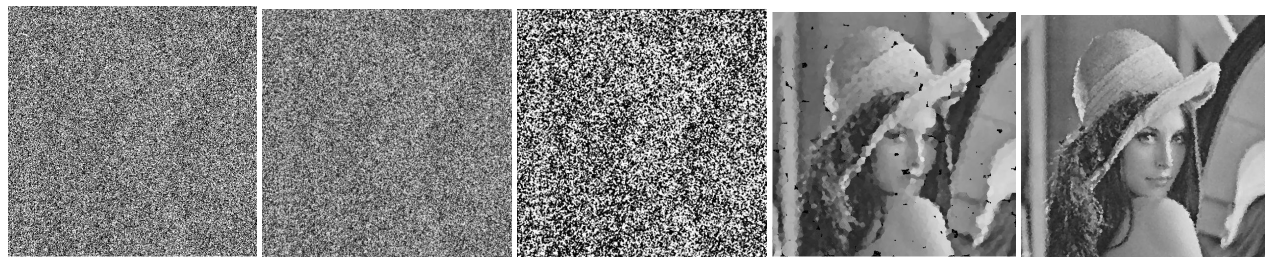

Figure 1. Salt and pepper noise reduction by Wiener, median, Sathua's and nbd filters with various noise levels: a) salt and pepper noise with variance $\sigma=0.25$; b) salt and pepper noise with variance $\sigma=0.5$; c) salt and pepper noise with variance $\sigma=0.75$; d) salt and pepper noise with variance $\sigma=0.9$

Figure 2 shows another example to prove the accuracy of nbd-filter. 


\begin{tabular}{lllll}
\hline Noisy image & Wiener filter & Median filter & Sathua's filter & nbd filter \\
\hline
\end{tabular}

a)
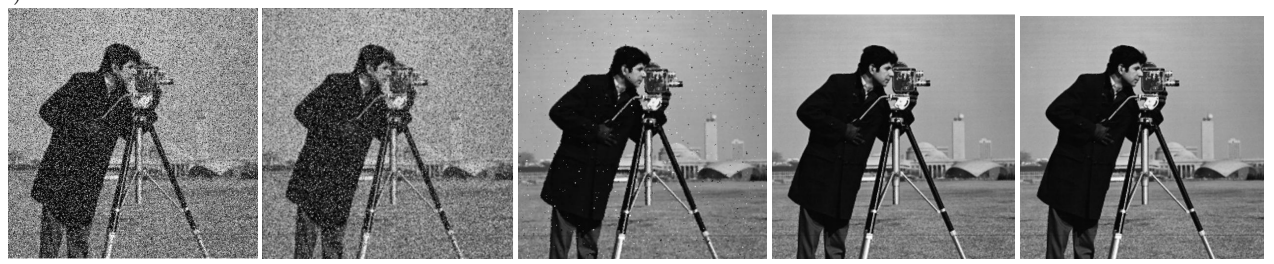

b)
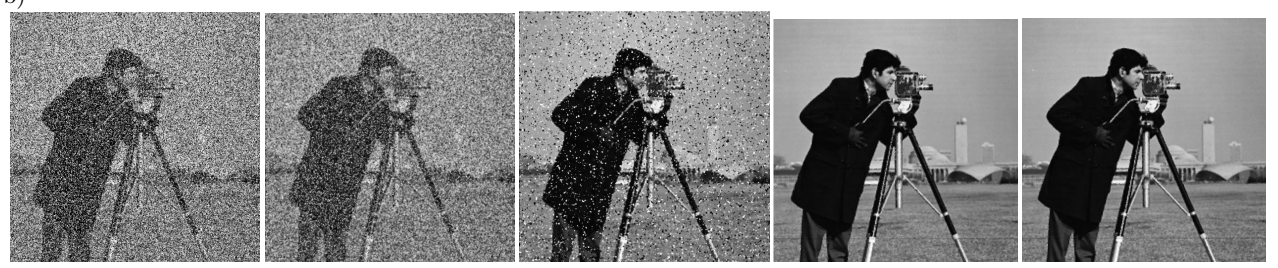

c)
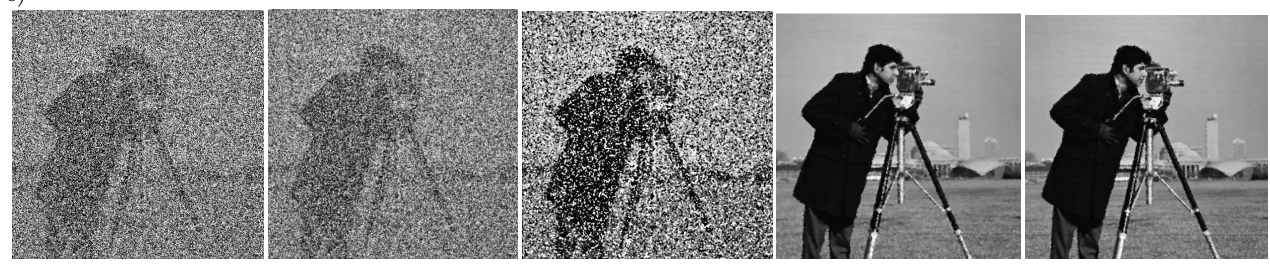

d)
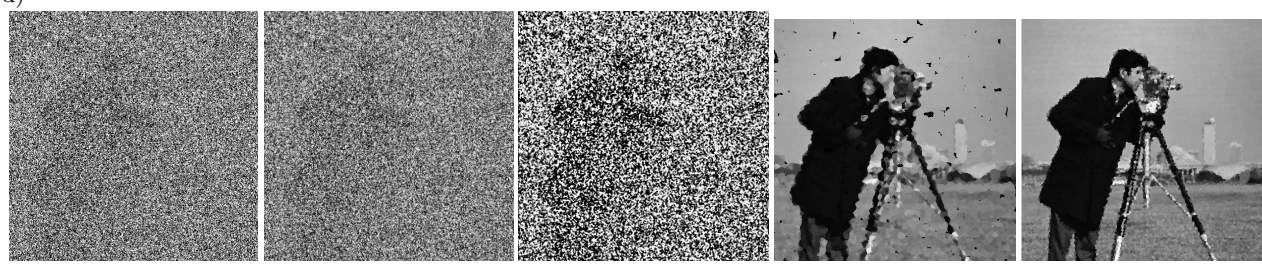

Figure 2. Salt and pepper noise reduction by Wiener, median, Sathua's and nbd filters with various noise levels: a) salt and pepper noise with variance $\sigma=0.25$; b) salt and pepper noise with variance $\sigma=0.5$; c) salt and pepper noise with variance $\sigma=0.75$; d) salt and pepper noise with variance $\sigma=0.9$

\subsection{Performance analysis}

The above images shows the efficiency of our proposed filter(nbd-filter). nbd-filter works very well at any level of noise intensity. Peak signal-to-Noise ratio (PSNR) is one of the tools to measure the quality of filtered image compared with the original image. Mean Square Error (MSE) determines the noise error of the filtered image. Following tables 1, 2, 3 and 4. shows the PSNR and MSE values of the Lena image and Cameraman image with the above-filtered images respectively. Following figures 3 and 4. represents the chart comparison of PSNR and MSE values of Lena image and Cameraman image by various filters such as mean, wiener, median, PTGMF and Sathua's method with proposed filter at different noise levels respectively. 
Table 1

PSNR Values of Lena image associated with filtered images

\begin{tabular}{|c|c|c|c|c|c|c|}
\hline Noise & \multicolumn{7}{|c|}{ PSNR Values } \\
\cline { 2 - 7 } level $(\sigma)$ & mean & wiener & median & PTGMF & Sathua's & nbd \\
\hline 0.1 & 23.771 & 23.523 & 32.880 & 42.662 & 42.829 & 42.8845 \\
\hline 0.2 & 20.737 & 18.041 & 28.546 & 39.016 & 38.846 & 39.024 \\
\hline 0.3 & 18.784 & 16.932 & 23.713 & 36.542 & 36.587 & 36.575 \\
\hline 0.4 & 17.278 & 16.008 & 18.816 & 34.451 & 34.529 & 34.731 \\
\hline 0.5 & 16.154 & 17.345 & 15.310 & 32.006 & 32.964 & 32.919 \\
\hline 0.6 & 15.055 & 14.452 & 12.316 & 28.800 & 31.298 & 31.575 \\
\hline 0.7 & 14.134 & 13.731 & 9.997 & 24.684 & 29.245 & 29.808 \\
\hline 0.8 & 13.310 & 13.046 & 8.123 & 20.113 & 26.821 & 27.557 \\
\hline 0.9 & 12.666 & 13.731 & 6.667 & 15.881 & 21.003 & 24.591 \\
\hline
\end{tabular}

Table 2

MSE Values of Lena image associated with filtered images

\begin{tabular}{|c|c|c|c|c|c|c|}
\hline \multirow{2}{*}{$\begin{array}{c}\text { Noise } \\
\text { level }(\sigma)\end{array}$} & \multicolumn{7}{|c|}{ MSE Values } \\
\cline { 2 - 7 } & mean & wiener & median & PTGMF & Sathua's & nbd \\
\hline 0.1 & 0.0042 & 0.0044 & 0.0005 & 0.0001 & 0.000052 & 0.000053 \\
\hline 0.2 & 0.0084 & 0.0157 & 0.0014 & 0.0001 & 0.000130 & 0.000125 \\
\hline 0.3 & 0.0132 & 0.0203 & 0.0043 & 0.0002 & 0.000219 & 0.000220 \\
\hline 0.4 & 0.0187 & 0.0251 & 0.0131 & 0.0004 & 0.000353 & 0.000336 \\
\hline 0.5 & 0.0242 & 0.0184 & 0.0294 & 0.0006 & 0.000505 & 0.000511 \\
\hline 0.6 & 0.0312 & 0.0359 & 0.0487 & 0.0013 & 0.000742 & 0.000696 \\
\hline 0.7 & 0.0386 & 0.0424 & 0.0710 & 0.0034 & 0.001190 & 0.001045 \\
\hline 0.8 & 0.0467 & 0.0496 & 0.0841 & 0.0097 & 0.002079 & 0.001755 \\
\hline 0.9 & 0.0541 & 0.0424 & 0.1054 & 0.0258 & 0.007937 & 0.003475 \\
\hline
\end{tabular}

Table 3

PSNR Values of Cameraman image associated with filtered images

\begin{tabular}{|c|c|c|c|c|c|c|}
\hline \multirow{2}{*}{$\begin{array}{c}\text { Noise } \\
\text { level }(\sigma)\end{array}$} & \multicolumn{7}{|c|}{ PSNR Values } \\
\cline { 2 - 7 } & mean & wiener & median & PTGMF & Sathua's & nbd \\
\hline 0.1 & 23.310 & 22.468 & 33.317 & 43.630 & 43.305 & 43.568 \\
\hline 0.2 & 19.954 & 17.224 & 27.988 & 39.097 & 39.220 & 39.606 \\
\hline 0.3 & 17.830 & 16.065 & 23.112 & 36.312 & 36.485 & 36.680 \\
\hline 0.4 & 16.278 & 16.052. & 18.602 & 34.125 & 34.336 & 34.620 \\
\hline 0.5 & 15.066 & 16.026 & 14.856 & 31.502 & 32.402 & 32.714 \\
\hline 0.6 & 13.794 & 13.265 & 11.938 & 27.853 & 30.178 & 30.793 \\
\hline 0.7 & 12.773 & 12.443 & 9.592 & 23.176 & 28.022 & 29.106 \\
\hline 0.8 & 11.969 & 11.764 & 7.757 & 18.814 & 25.430 & 26.796 \\
\hline 0.9 & 11.144 & 11.028 & 6.239 & 14.408 & 20.302 & 23.610 \\
\hline
\end{tabular}


Table 4

MSE Values of Cameraman image associated with filtered images

\begin{tabular}{|c|c|c|c|c|c|c|}
\hline \multirow{2}{*}{$\begin{array}{c}\text { Noise } \\
\text { level }(\sigma)\end{array}$} & \multicolumn{7}{|c|}{ MSE Values } \\
\cline { 2 - 7 } & mean & wiener & median & PTGMF & Sathua's & nbd \\
\hline 0.1 & 0.0047 & 0.0057 & 0.0005 & 0.0001 & 0.000047 & 0.000044 \\
\hline 0.2 & 0.0101 & 0.0190 & 0.0016 & 0.0001 & 0.000120 & 0.000110 \\
\hline 0.3 & 0.0165 & 0.0247 & 0.0049 & 0.0002 & 0.000225 & 0.000215 \\
\hline 0.4 & 0.0236 & 0.0312 & 0.0138 & 0.0004 & 0.000368 & 0.000345 \\
\hline 0.5 & 0.0311 & 0.0250 & 0.0327 & 0.0007 & 0.000575 & 0.000535 \\
\hline 0.6 & 0.0417 & 0.0471 & 0.0540 & 0.0016 & 0.000960 & 0.000833 \\
\hline 0.7 & 0.0528 & 0.0570 & 0.0700 & 0.0048 & 0.001577 & 0.001229 \\
\hline 0.8 & 0.0636 & 0.0666 & 0.0876 & 0.0131 & 0.002865 & 0.002091 \\
\hline 0.9 & 0.0768 & 0.0789 & 0.1177 & 0.0362 & 0.009329 & 0.004355 \\
\hline
\end{tabular}
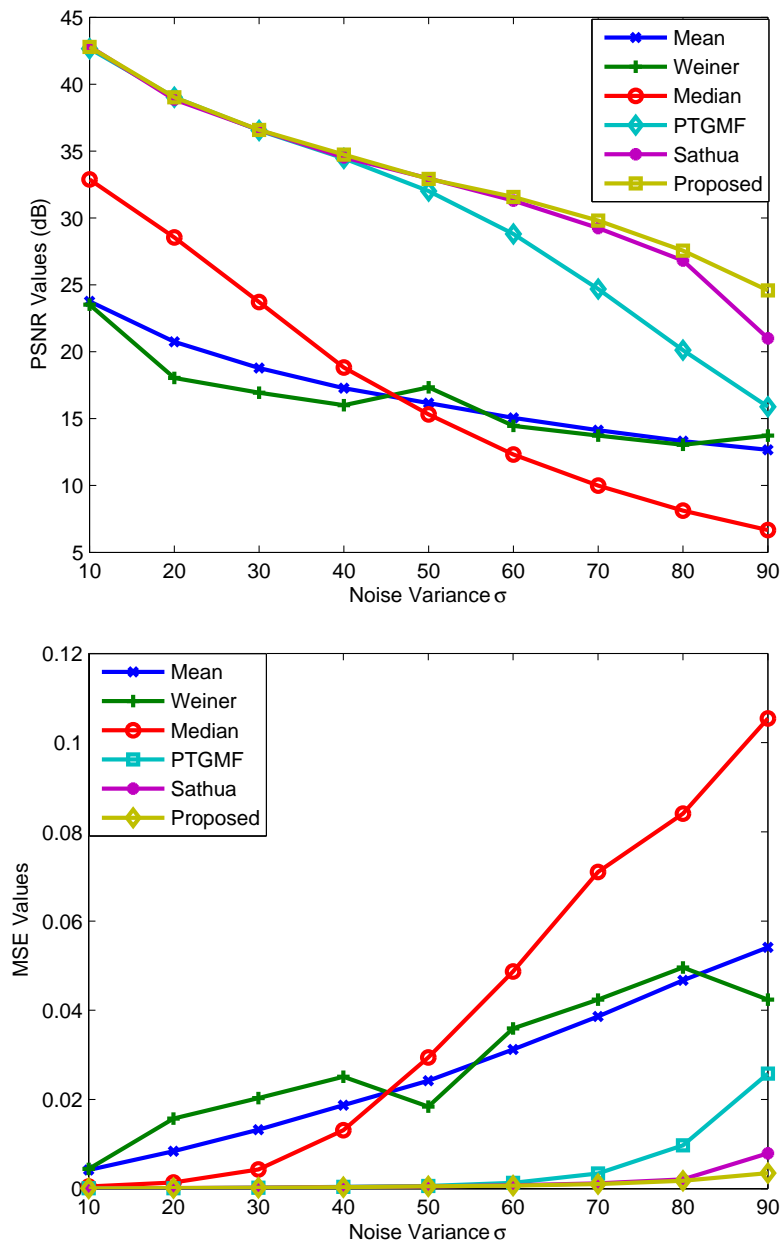

Figure 3. PSNR and MSE comparison of lena image with filtered image 

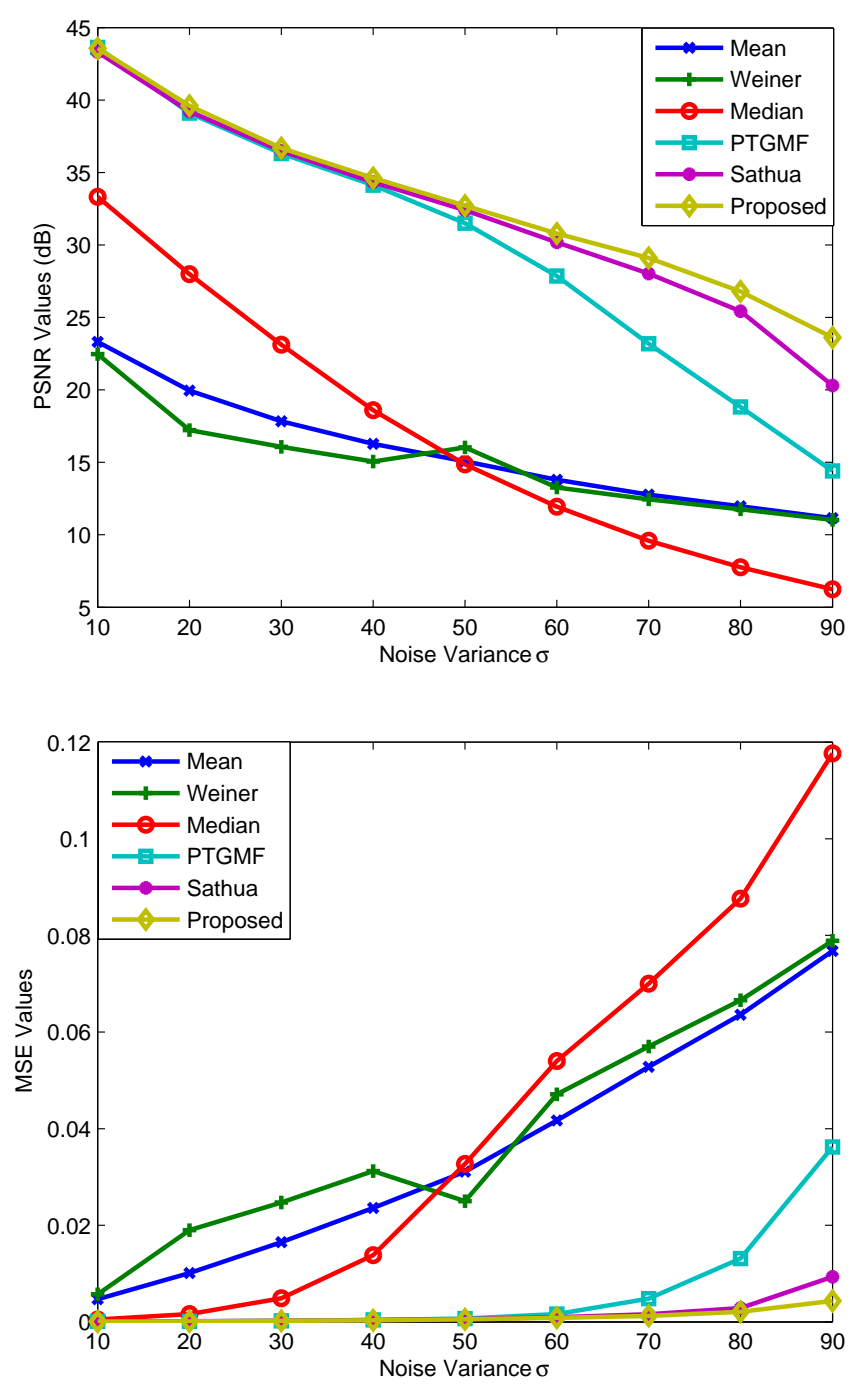

Figure 4. PSNR and MSE comparison of cameraman image with filtered image

\subsection{Edge detection results}

In this section, we compare our edge detector with most popular edge detectors Canny, Roberts, Sobel, and Prewitt. Figure 5 shows the edge detected at different salt and pepper noise levels along with nbd-filter by our proposed edge detector.

We can see clear edges of sample images regardless of noise levels. 

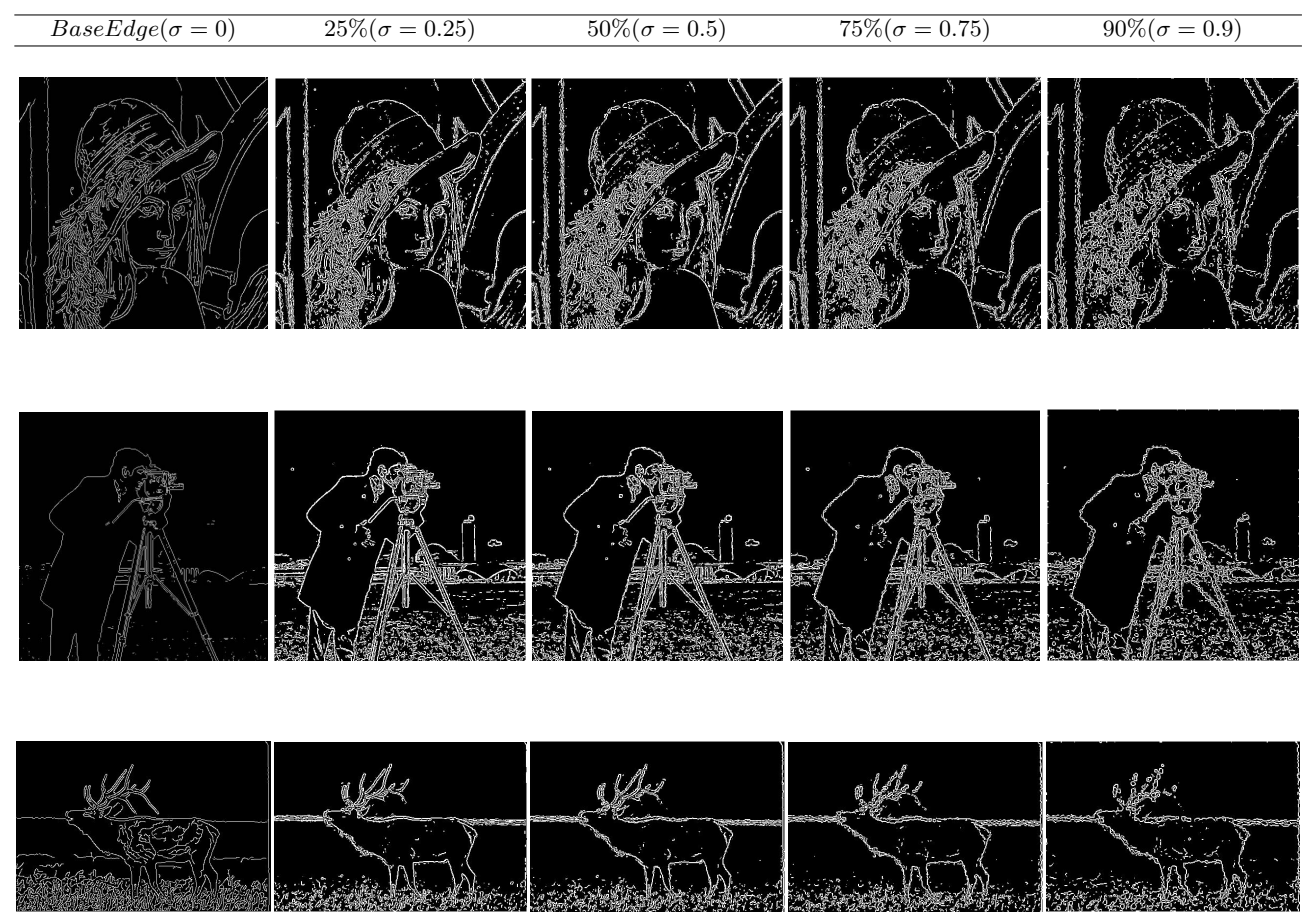

Figure 5. Edge detected images at different noise levels $(\sigma)$

Proposed edge detector works perfectly for noise-free images also. It detects edges significantly compared to most of the other edge detectors. Sample images of Lena, cameraman, coins, house, and dog are given below (Fig. 6).

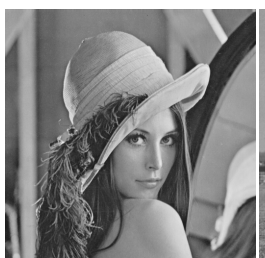

Lena

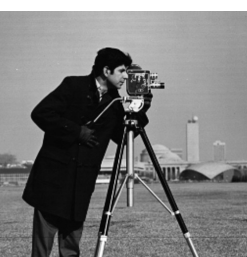

Cameraman

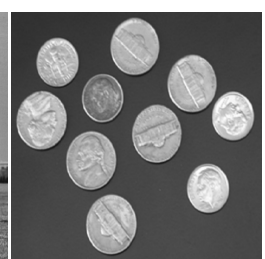

coins

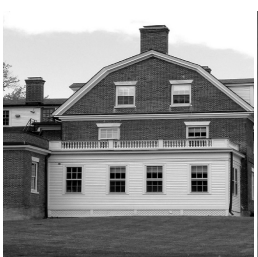

house

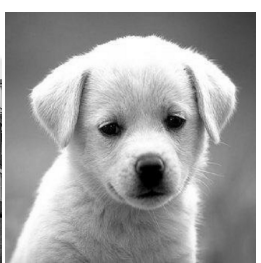

Dog

Figure 6. Orignal images

Figure 7 shows the edge detection of the above sample images by using various detectors. 


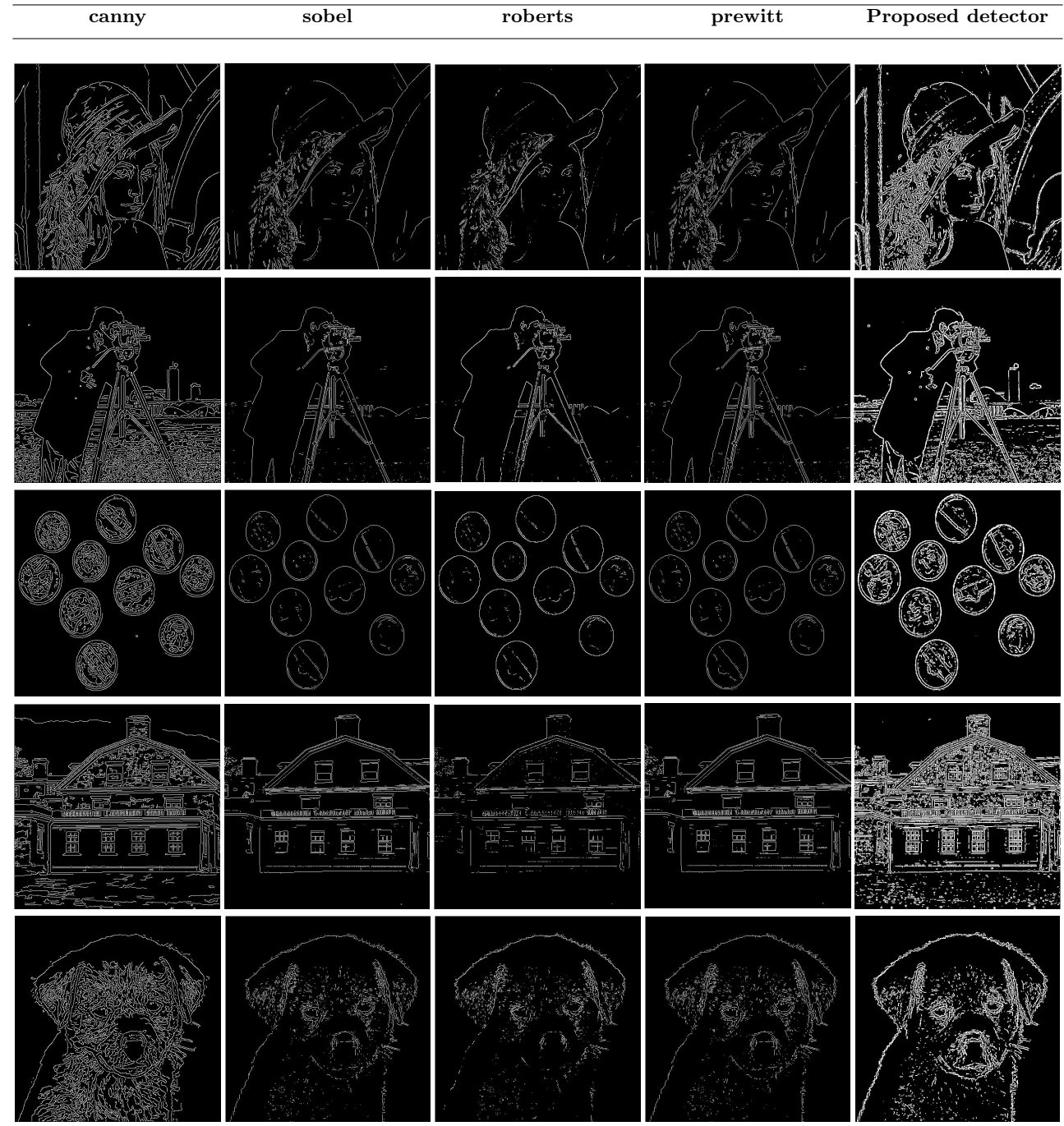

Figure 7. Edge detection by canny, sobel, roberts, prewitt and proposed detectors

\subsection{Performance analysis}

In this section, we analyze the edge detection results by Structural Similarity Index Measurement (SSIM) and Edge based Structural Similarity Index Measurement(ESSIM). Structural Similarity Index Measurement was proposed by Zhou Wang [16] in 2004, which extracts image structural information based on their parameters such as luminance(l), contrast(c) and structure(s). SSIM is defined as

$$
S S I M=[l(x, y), c(x, y), s(x, y)]
$$


Table 5 shows the SSIM values of various edge detected images.

Table 5

Structural Similarity Index Measure values

\begin{tabular}{|c|c|c|c|c|c|}
\hline \multirow{2}{*}{$\begin{array}{c}\text { Edge } \\
\text { Image }\end{array}$} & \multicolumn{5}{|c|}{ SSIM Values } \\
\cline { 2 - 6 } & Sobel & Roberts & Prewitt & Canny & Proposed \\
\hline Lena & 0.0467 & 0.0457 & 0.0466 & 0.0886 & 0.1246 \\
\hline Cameraman & 0.8000 & 0.8003 & 0.8000 & 0.8621 & 0.8894 \\
\hline Coins & 0.0978 & 0.0985 & 0.0978 & 0.1300 & 0.1313 \\
\hline House & 0.0594 & 0.0579 & 0.0595 & 0.0944 & 0.1286 \\
\hline Dog & 0.5386 & 0.5365 & 0.5386 & 0.5822 & 0.5941 \\
\hline
\end{tabular}

Chen [2] proposed Edge based structural similarity index for image quality assessment in 2006; it is an another edge quality assessment for images. ESSIM compares the edge information between original image and distorted image.

$$
\operatorname{ESSIM}=[l(x, y), c(x, y), e(x, y)]
$$

Table 6 shows the ESSIM values of various edge detected images.

Table 6

Edge based Structural Similarity Index Measure Values

\begin{tabular}{|c|c|c|c|c|c|}
\hline \multirow{2}{*}{$\begin{array}{c}\text { Edge } \\
\text { Image }\end{array}$} & \multicolumn{5}{|c|}{ ESSIM Values } \\
\cline { 2 - 6 } & Sobel & Roberts & Prewitt & Canny & Proposed \\
\hline Lena & 0.9921 & 0.9921 & 0.9921 & 0.9925 & 0.9927 \\
\hline Cameraman & 0.9915 & 0.9915 & 0.9915 & 0.9919 & 0.9921 \\
\hline Coins & 0.9937 & 0.9937 & 0.9937 & 0.9940 & 0.9942 \\
\hline House & 0.9875 & 0.9874 & 0.9875 & 0.9879 & 0.9882 \\
\hline Dog & 0.9940 & 0.9940 & 0.9940 & 0.9944 & 0.9945 \\
\hline
\end{tabular}

Above SSIM and ESSIM values shows the performance of the proposed edge detector.

\section{Conclusion}

In this paper, a novel image denoising technique called neighborhood filter (nbd-filter) is proposed to reduce Salt and pepper noise. Further, an edge detection technique for grayscale images based on neutrosophic set is proposed. The experimental results shows the performance of the proposed denosing filter and the proposed edge detector. The performance analysis section shows the efficiency of the proposed filter and edge detector over the conventional techniques. The proposed nbd-filter gives much better result than conventional filters. However the proposed filter has its own limitations such as it is only suitable for images which corrupted by Salt and pepper noise and also 
less effective on binary images. The proposed edge detector performed well on noise and noise-free grayscale images. We expect that these denoising and edge detection techniques will achieve more applications in image processing.

\section{References}

[1] Arulpandy P., Pricilla M.T.: Reduction of indeterminacy of grayscale image in bipolar neutrosophic domain, Neutrosophic Sets and Systems, vol. 28(1), 2019. https://doi.org/10.5281/zenodo.3382501.

[2] Chen G.-H., Yang C.-L., Po L.-M., Xie S.-L.: Edge-Based Structural Similarity for Image Quality Assessment. In: 2006 IEEE International Conference on Acoustics Speech and Signal Processing Proceedings, vol. II, pp. 933-936, 2006. http://dx.doi.org/10.1109/ICASSP.2006.1660497.

[3] Cheng H.D., Guo Y.: A new neutrosophic approach to image thresholding, New Mathematics and Natural Computation, vol. 4(3), pp. 291-308, 2008.

[4] Cheng H.D., Guo Y., Zhang Y.: A novel image segmentation approach based on neutrosophic set and improved fuzzy C-means algorithm, New Mathematics and Neural Computation, vol. 7(1), pp. 155-171, 2011. https://doi.org/10.1142/S179 3005711001858.

[5] Guo Y., Cheng H.D., Zhang Y.: A new neutrosophic approach to image denoising, New Mathematics and Neural Computation, vol. 5(3), pp. 653-662, 2009. https: //doi.org/10.1142/S1793005709001490.

[6] Guo Y., Şengür A.: A novel image edge detection algorithm based on neutrosophic set, Computers and Electrical Engineering, vol. 40(8), pp. 3-25, 2014. https://doi.org/10.1016/j.compeleceng.2014.04.020.

[7] Ma H., Nie Y.: A two-stage filter for removing salt-and-pepper noise using noise detector based on characteristic difference parameter and adaptive directional mean filter, PLOS ONE, vol. 13(10), 2018. https://doi.org/10.1371/journal.pone .0205736 .

[8] Mohan J., Krishnaveni V., Guo Y.: Performance analysis of neutrosophic set approach of median filtering for MRI denoising, International Journal of Electronics and Communication Engineering and Technology, vol. 3(2), pp. 148-163, 2012.

[9] Pratt W.K.: Introduction to Digital Image Processing, Taylor and Francis Group, Boca Raton, 2013. https://doi.org/10.1201/b15731.

[10] Raza M.T., Sawant S.: High density salt and pepper noise removal through decision based partial trimmed global mean filter. In: Engineering (NUiCONE), 2012 Nirma University International Conference, pp. 1-5, 2012.

[11] Sathua S.K., Dash A., Behera A.: Removal of Salt and Pepper noise from GrayScale and Color Images: An Adaptive Approach, International Journal of Computer Science Trends and Technology, vol. 5(1), pp. 117-126, 2017. 
[12] Sert E., Alkan A.: Image edge detection based on neutrosophic set approach combined with Chan-Vase algorithm, International Journal of Pattern Recognition and Artificial Intelligence, vol. 33(3), 2019. https://doi.org/10.1142/S02180 01419540089 .

[13] Smarandache F.: Neutrosophy: A new branch of philosophy, Multiple Valued Logic: An International Journal, vol. 8, pp. 297-384, 2002.

[14] Smarandache F.: A unifying field in logics: neutrosophic logic. Neutrosophy, neutrosophic set, neutrosophic probability and statistics, 3rd edition, American Research Press, 2003.

[15] Smarandache F.: Neutrosophic Set - A Generalization of the Intituitionistic Fuzzy Set, International Journal of Pure and Applied Mathematics, vol. 24(3), pp. 287-297, 2005.

[16] Wang Z., Bovik A.C., Sheikh H.R., Simoncelli E.P.: Image Quality Assessment: From Error Visibility to Structural Similarity, IEEE Transactions on Image Processing, vol. 13(4), pp. 600-612, 2004.

\section{Affiliations}

\section{P. Arulpandy}

Department of Mathematics, Bannari Amman Institute of Technology, Erode, Tamilnadu, India, arulpandy002@gmail.com, ORCID ID: https://orcid.org/0000-0001-9876-8509

\section{Trinita Pricilla}

Department of Mathematics, Nirmala College for Women, Coimbatore, Tamilnadu, India, abishai_kennet@yahoo.in

Received: 26.09 .2019

Revised: 27.03.2020

Accepted: 03.04.2020 